\title{
OBSERVABLE SIGNALS OF CORONAL HEATING PROCESSES
}

\author{
P. VENKATAKRISHNAN \\ Space Science Laboratory \\ Marshall Space Flight Center, Al 35812, U.S.A.
}

Abstract. The solar corona is thought to be sustained by waves, currents, turbulence or by velocity filtration. For efficient wave heating of the corona, only the Alfven waves seem to survive the effects of steepening and shock dissipation in the chromosphere (Zirker, 1993, Solar Phys. 148,43) and these can be dissipated in the corona by mode conversion or phase mixing (Priest, 1991 in XIV Consultation on Solar Physics, Karpacz). Enhanced line width of $530.3 \mathrm{~nm}$ coronal line seen within closed structures (Singh $e t$ al., 1982, J. Astrophys. Astron. 3,248 ), association of enhanced line width of HeI $1083 \mathrm{~nm}$ line with enhanced equivalent width (Venkatakrishnan et al., 1992, Solar Phys. 138,107 ), and gradients seen in the MgX 60.9 and $62.5 \mathrm{~nm}$ coronal line width (Hassler, et al., 1990, Astrophys. J. 348,L77), are possibly some examples of the observed signals of wave heating. Current sheets, produced in a variety of ways (Priest and Forbes, 1989, Solar Phys. 43,177; Parker, 1979, Cosmical Magnetic Fields, Ox. Univ. Press ), can dissipate and provide heat. The properties of current sheets can be inferred from fill factors, emission measures (Cargill, 1994, in J.L. Burch and J.H. Waite, Jr. (eds.) Solar System Plasma Physics: Resolution of Processes in Space and Time, AGU Monograph ), hard xrays (Lin et al., 1984, Astrophys. J. 283,421), and radio bursts (Benz, 1986, Solar Phys. 104,99 ). The association of large scale currents with enhanced transition region (deLoach et al., 1984, Solar Phys. 91,235. ) and regions of enhanced magnetic shear with brighter corona (Moore et al., 1994, Proc. Kofu Symp) are of some possible interest in this context. Self consistent calculations of the turbulent cascade of energy from the scales of photospheric motions down into dissipative scales (Heyvaerts and Priest, 1992, Astrophys. J. 390 , 297 ) predict the width of coronal lines as a function of the properties of the forcing flows. Velocity filtration caused by free streaming effects off a non maxwellian boundary distribution of particles may well result in a plasma having coronal properties (Scudder, 1992a, Astrophys.J. 398,299; 

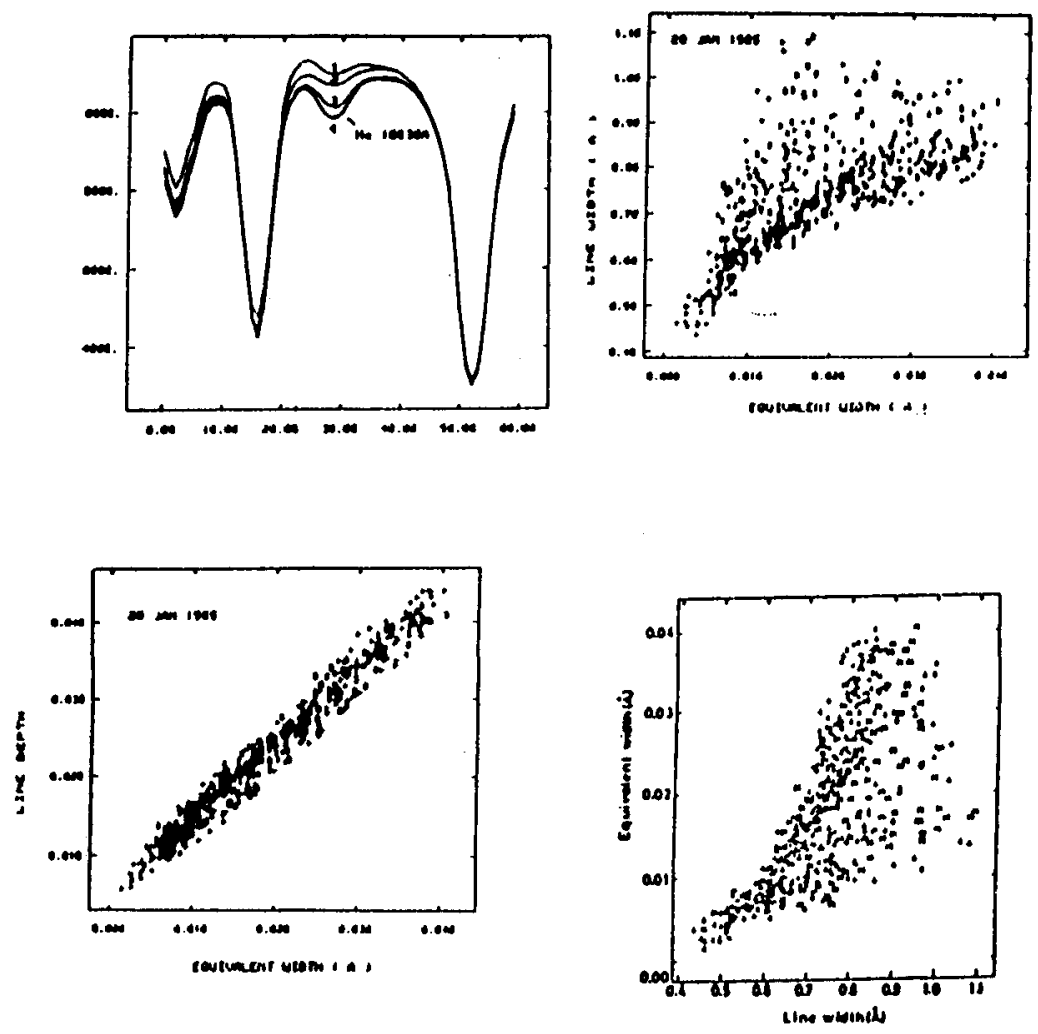

Figure 1. Top left panel shows the spectra in the vicinity of HeI $1083 \mathrm{~nm}$. Top right panel is line width (in Angstroms) plotted against equivalent width (in Angstrom). Bottom left panel is central line depth plotted against equivalent width.

1992b, Astrophys.J. 398,319). The observable signals are the variation of line shapes with altitude.

This talk focusses attention on the observed properties of the HeI 1083 $\mathrm{nm}$ line parameters as possible signatures of a heating process. The data consisted of a time series of HeI $1083 \mathrm{~nm}$ spectra from the solar disc, outside of active regions. The time averages of the line parameters like equivalent width and line width, for the various spatial positions on the disc were plotted against each other (figure 1). These plots can be used to imply that the coronal enhancements are related to enhancements in the underlying chromospheric energy density (Venkatakrishnan, 1993, Solar Phys. 148,233 ), thereby signalling either wave heating or velocity filtration.

This talk is dedicated to the late Professor M.K.V. Bappu. 